\title{
Dynamics and planet formation in/around binaries
}

\author{
F. Marzari ${ }^{1}$ P. Thébault ${ }^{2,3}$ S. Kortenkamp ${ }^{4}$ and H. Scholl ${ }^{5}$ \\ 1 Dipartimento di Fisica, Via Marzolo 8, 35131 Padova, Italy marzari@pd.infn.it \\ 2 Observatoire de Paris, Section de Meudon, F-92195 Meudon Principal Cedex, \\ France philippe.thebault@obspm.fr \\ 3 Stockholm Observatory, Albanova Universitetcentrum, SE-10691 Stockholm, \\ Sweden thebault@astro.su.se \\ 4 Planetary Science Institute, Tucson, Arizona kortenka@psi.edu \\ ${ }^{5}$ Observatoire de la Côte d'Azur, Nice, France scholl@obs-nice.fr
}

\section{Summary.}

We study to which extent planetesimal accretion is affected by the perturbing presence of a compagnon star. We concentrate on one crucial parameter: the distribution of encounter velocities within the planetesimal swarm. We numerically explore the evolution of this parameter taking into account the secular perturbations of the binary and friction due to the very likely presence of gas in the disk. $\langle\Delta v\rangle$ maps are derived, for planetesimals of different sizes, for a total of 120 binary configurations (eccentricity eb and separation ab). We identify for each case 3 different accreting behaviours. 1) In regions where no significant dV increase is observed, "classical" (i.e., single-star) accretion is possible. 2) In regions where $d V>v_{\text {ero }}$, the threshold velocity above which all impacts are eroding, no accretion is possible and planet growth is stopped. 3) In between these 2 limiting behaviours, a large fraction of binary configurations leads to significant $\mathrm{dV}$ increase, but still below the erosion threshold. In this intermediate case, planetesimal growth can occur, but proceeds slower than in the single-star case, possibly following the so-called type II runaway groth mode.

\section{Introduction}

\section{1 the "standard" planet formation scenario}

A fundamental step in the "standard" scenario for the formation of planets, either terrestrial or gaseous giant, is the collisional accumulation of kilometersized planetesimals in successively larger bodies. It is widely admitted that planetesimals emerge from the coagulation of silicate and ice grains in the midplane of protoplanetary disks on timescales of the order of $10^{4} \mathrm{yrs}$, even if the details of the formation process are not well understood at present. Colliding with each other, planetesimals grow into large planetary embryos 
(Lunar- to Mars-size) through a phase of "runaway" and "oligarchic" growth on a timescale of the order of $10^{4}-10^{5}$ years (Greenberg et al., 1978; Wetherill \& Stewart, 1989; Barge \& Pellat, 1993; Lissauer, 1993; Kokubo \& Ida, 1998, 2000; Rafikov, 2003, 2004). This is a robust finding corroborated by distinct numerical methods (see review by Kortenkamp et al., 2000). For terrestrial planets, the formation process reaches completion after a final phase of giant impacts and mutual accretion of planetary embryos resulting in $10^{27}-10^{28}$ gram full-size bodies in about $10^{7}-10^{8}$ years on stable separated orbits (Chambers and Wetherill, 1998). In the case of giant planets, protoplanets accumulate into a solid core that, once sufficiently massive, begins to accrete a gaseous envelope. Initially, this envelope is in hydrostatic equilibrium sustained by the luminosity provided by the accreting planetesimals and is increasing at a slow rate compared to that of the solid core. However, at a critical core mass (in between 5 to $15 M_{\oplus}$ ) a phase of runaway gas accretion occurs, the planet mass increases rapidly and it reaches its final value on a timescale estimated to be only a few $10^{3}$ yr. For a given density of solids and gas in the disk, hydrodynamical calculations can determine for which mass of the core the envelope is able to remain static or collapse, and the rate at which the mass flows onto the planet (see e.g. Wuchterl et al., 2000). At the end of the gas rapid infall, the planet evolves into the isolated stage during which it cannot grow any further because of the exaustion of the gas supply either because of its removal by effects of the star or as a result of gap formation around the planet orbit triggered by disk-planet tidal interaction (Papaloizou and Lin, 1984). The planet finally cools down and contracts to its present size. This scenario for giant planet formation, called core accretion (Pollack et al, 1996), is based on the interior models for Jupiter and Saturn which strongly suggest the presence of a dense central core surrounded by an envelope composed mainly by hydrogen and helium for both planets. There are large uncertainties on the mass of the core and detailed calculations performed by (e.g. Guillot, 1999) predict un upper limit of $10 M_{\oplus}$ for Jupiter's core while for Saturn the core mass ranges from 6 to $17 M_{\oplus}$.

It is believed that extrasolar planets, detected in orbit around an increasing number of nearby stars may have formed either by core accretion or by disk instability (e.g. Boss, 1997). While core accretion requires a few million years to form a gas giant planet, in the competing model self-gravitating clumps formed by disk instability will contract to planetary densities in times of a few hundred years. The two formation mechanisms are both viable and, at the moment, there is no reason to definitively exclude one in favor of the other. In this paper, however, we will concentrate on planetesimal accretion as a common mechanism for forming either terrestrial planets or the core of gaseous giants.

In a given protoplanetary disk, the potentiality of a planetesimal swarm to accumulate into larger bodies is measured by two critical parameters. 
- The surface density of solid material. The concentration of condensible material determines the final mass in the case of a terrestrial planet, while it must lead to a reasonable formation time for the core of a giant planet that must be compatible with the lifetime of the gaseous disk. Disk ages are estimated to range from 0.1 to $10 \mathrm{Myr}$ (Strom et al., 1993; Haisch et al., 2001; Chen and Kamp, 2004), while 3 Myr is the age at which half of the stars show evidence of disks. Simulations of the accumulation of jovian planets (Bodenheimer and Lin, 2002) show that if the surface density of solids in the disk is assumed to be at least 3-times larger than that of the minimum-mass solar nebula (Weidenschilling, 1977) then the formation time for Jupiter mass planets is within 2-3 Myr at $5 \mathrm{AU}$ from the star. If planetary migration is included in the simulations, the timescale for forming Jupiter is reduced to 1-2 Myr even with solid surface density closer to the minimum mass solar nebula (Rice and Armitage, 2003).

- The relative encounter velocities between planetesimals.

The random encounter velocities in a planetesimal swarm determine whether mutual impacts will result in accretion or on the contrary in cratering or fragmentation. The key mechanisms controlling the impact velocities are: 1) mutual gravitational encounters between planetesimals, 2) physical collisions, 3) gas drag, in presence of the gaseous component of the disk. Several numerical studies have shown that in an unperturbed swarm of kilometer-sized planetesimals around a single star the average random encounter velocity $\langle\Delta v\rangle$ is low enough to favor the collisional accumulation of bodies (e.g. Greenberg et al., 1978). At the early stages of accretion a low $\langle\Delta v\rangle$ is also a fundamental requirement for permitting a phase of rapid "runaway" growth which significantly shortens the timescale for the formation of planetary embryos. Indeed, under this condition the ratio $v_{e s c(R)} /\langle\Delta v\rangle$ between the escape speed of the largest bodies and the average impact velocities is $>>1$, thus making their gravitational crosssection quite larger than the geometrical cross-section. This gravitational focusing enhances the growth rate of the larger planetesimals which grow much faster than the rest of the population (e.g. Lissauer, 1993). A direct consequence of this crucial $\langle\Delta v\rangle$ dependence is that planetesimal growth is very sensitive to any increase of the encounter velocities. It could thus be significantly slowed down or even stopped if the gravitational influence of a massive body external to the swarm, like a a pre-existing giant planets (e.g., Jupiter in our solar system) or a companion star in a binary system is able to excite large impact velocities. Thébault et al. (2002) have shown that the formation of terrestrial planets in those exoplanetary systems hosting a giant planet on an external orbit may have been indeed halted. They find that in both the $\epsilon$ Eridani and 47 Uma planetary systems, the relative velocity between planetesimals in the terrestrial planet region may have quickly exceed the critical threshold value $v_{\text {ero }}$ beyond which erosion dominates before the completion of the runaway growth if the massive outer planet rapidly reached its present mass. Of course, the 
threshold velocity that preferentially result in disruption rather than accretion is a sensitive function of the internal strength of planetesimals, their bulk density and the fraction of impact energy partitioned into the fragments after the impact. These physical parameters strongly depend on the initial radial density and temperature profile in the disk midplane.

\section{2 planets in binaries, planet formation in binaries}

In this paper we will focus on how the presence of a companion star affects the planetesimal accretion process either in the formation of terrestrial planets or the core of a giant planet. While the existence of extrasolar Earth-like planets has yet to be assessed, some of the giant exoplanets (up to about 40 so far) have been detected in the so-called S-type orbits that encircle one component of a binary system (e.g. Raghavan et al., 2006; Desidera \& Barbieri, 2007). Most of these are widely separated pairs where the stellar companion probably does not have a significant influence on the planetesimal accretion around the other star. However, in three of the systems, $\gamma$ Cephei GL 86, and HD41004, the companion is within 20-25 AU from the star hosting the planet. The $\gamma$ Cephei system is made of a central star with an estimated mass of $1.40 \pm 0.12 M_{\odot}$, a planet orbiting at $2.13 \pm 0.05 \mathrm{AU}$ with a minimum mass of $1.6 \pm 0.13 M_{J}$ and a companion star on an eccentric orbit $(e=0.411 \pm 0.0063)$ with semimajor axis of 20.23 $\pm 0.64 \mathrm{AU}$ and mass of about $0.4 M_{\odot}$ (Hatzes et al., 2003; Torres, 2007; Neuhäuser et al., 2007). GL 86 is a dwarf star somewhat less massive than the Sun (about $0.7 M_{\odot}$, e.g. Santos et al., 2004) with a white dwarf companion of $0.55 M_{\odot}$ (Mugrauer and Neuhauser, 2005) on an orbit having a semimajor axis of 18.42 AU and an eccentricity of e=0.3974 (Lagrange et al., 2006). A massive jovian planet $\left(M \sin (i)=3.91 M_{J}\right)$ moves around the primary star on an almost circular orbit $(e=0.0416)$ with a semimajor axis of $a=0.113 \mathrm{AU}$ (Eggenberger et al., 2003). HD41004 A is a $0.7 M_{\odot}$ star with a dwarf M4V companion orbiting at $\simeq 22 A U$. The primary star has a $M \sin (i)=2.3 M_{J}$ planetary companion on a very eccentric $\mathrm{e}=0.39$ orbit at $a=1.31 \mathrm{AU}$ (Zucker et al., 2004; Raghavan et al., 2006). Note that this system is a hierarchical quadruple, since the stellar companion is itself orbited at $a=0.016 \mathrm{AU}$ by a brown dwarf of minimum mass $18.4 M_{J}$ (Zucker et al., 2004).

The observed characteristics of these systems, e.g., the proximity of the companion star and its eccentric orbit, strongly suggest that the presence of the secondary star must have had an influence on the formation of the detected planets. The gravitational pull of the companion star acts on the $\langle\Delta v\rangle$ of the planetesimal disk around the primary star. As previously mentioned, this can modify the course of the accretion process which strongly depends on the ratio $v_{e s c} /\langle\Delta v\rangle$, where $v_{e s c}$ is the surface escape velocity of the biggest accreting bodies. Timing is an important issue since the perturbations will be effective in altering the planetesimal formation process only if $t_{s e c}$, the timescale required by the the secular perturbations of the star to induce a significant $\Delta v$ increase, is shorter than $t_{\text {grow }}$, the timescale for the runaway/oligarchic formation of 
embryos which is typically of the order of $10^{4}$ to $10^{5} \mathrm{yrs}$. We can outline three possible different evolutions for a perturbed planetesimal swarm:

- 1) If $\langle\Delta v\rangle$ remains smaller than $v_{e s c}$ during the growth phase then Planetary formation proceeds almost unaffected by the companion perturbations . The "classical" planetesimal accumulation scenario holds.

- 2) For values of $\langle\Delta v\rangle$ larger than $v_{e s c}$ but still smaller than $v_{e r o}$, the threshold velocity for erosion to dominate over accretion, planetary accretion will still be possible but runaway growth will either not occur or will occur only after large enough bodies have formed such that $\left.v_{\text {esc }}\right\rangle\langle\Delta v\rangle$ (the so-called Type II runaway growth described in Section 6 below).

- 3) If $\langle\Delta v\rangle$ is increased beyond $v_{e r o}$, in the majority of collisions cratering and fragmentation will overcome accretion and the planetesimal population will be slowly ground down to dust, failing to form a full size planet.

In the following, we will focus on the determination of $\langle\Delta v\rangle$ for different parameters of the binary star system with the goal of evaluating when planetesimal accretion is possible. We have performed a series of deterministic numerical simulations, following the dynamical evolution of a swarm of test planetesimals, under the influence of the companion star's gravitational pull and friction by the gas in which the planetesimals are imbedded. Crucial parameters, such as the companion star's mass $m_{b}$, semi-major axis $a_{b}$ and orbital eccentricity $e_{b}$ are explored as free parameters. We use a code initially developed for the study of planetesimal systems perturbed by giant planets (Thébault \& Brahic, 1998; Thébault \& Beust, 2001; Thébault et al., 2002) and adapted to the circumprimary case (Marzari \& Scholl, 2000; Thébault et al., 2004, 2006). This 3-D code has a close encounter tracking algorithm which enables precise determination of the $\Delta v$ within the system.

In a first step we present a detailed study of the planetesimal secular dynamics in presence of a companion star (section 2). We will then proceed by introducing the effects of gas drag and by estimating how the combined action of the friction force with the secular perturbations affect the value of $\langle\Delta v\rangle$ (section 3). We then compare the derived $\langle\Delta v\rangle$ with an an approximate estimate of $v_{\text {ero }}$ to derive a yes-or-no criterion for planetary formation as a function of the binary orbital parameters (section 4). Finally, in section 5, we present and describe one possible accretion-growth mode for planetesimals in perturbed binary star systems, the so-called Type II runaway growth mechanism.

\section{Planetesimal dynamics in a binary star system: the secular approximation}

Hereinafter, we will assume that the orbit of the secondary star is coplanar to the planetesimal disk so that we can neglect the inclination of the bodies. 
This is a reasonable assumption when the star is close to the primary because of the short relaxation time of the disk into the plane of the binary orbit.

Heppenheimer (1978) developed a simplified theory for the evolution of the planetesimal eccentricity with time based on an expansion of the disturbing function in a power series of the ratio of the semi-major axes $a / a_{b}$ where $a$ is the semimajor axis of the planetesimal and $a_{b}$ that of the companion star. The approximation holds if $a$ is small compared to $a_{b}$ and it stays within the critical semimajor axis $a_{c}$ for dynamical stability derived using direct numerical integrations by Wiegert and Holman (1997). By truncating the disturbing function of the secondary star at the second order in the eccentricity of the planetesimal, the secular equations simplify and lead to the following expressions for the forced eccentricity $e_{F}$ and the frequency $u$ of circulation of the perihelion $\varpi$ :

$$
\begin{gathered}
e_{F}=\frac{5}{4} \frac{a}{a_{b}} \frac{e_{b}}{1-e_{b}^{2}} \\
u=\frac{3}{4} \pi \frac{1}{\left(1-e_{b}^{2}\right)^{3 / 2}} m_{b} \frac{a^{3 / 2}}{a_{b}^{3}}
\end{gathered}
$$

with

$$
\tan (\varpi(t))=-\frac{\sin (2 u t)}{1-\cos (2 u t)}
$$

However, Thebault et al. (2006) compared the predictions of the above equations with direct numerical simulations and found significant discrepancies, especially for the oscillation frequency $u$. They empirically derived a revised expression for the frequency $u$ which reads:

$$
u=\frac{3}{4} \pi \frac{1}{\left(1-e_{b}^{2}\right)^{3 / 2}} m_{b} \frac{a^{3 / 2}}{a_{b}^{3}}\left[1+32{\frac{m_{b}}{1-e_{b}^{2}}}^{3}\left({\frac{a}{a_{b}}}^{2}\right)\right]
$$

The discrepancy between the numerical and analytical estimate of $u$ is reduced from about $70 \%$, in most of the cases with high $e_{b}$ and $e$, to less than $5 \%$.

To illustrate the dynamical evolution of a planetesimal swarm under the secular perturbations of a companion star we have performed a "pedagogical" numerical simulation where we integrate the orbits of massless test bodies, initially on circular oribts, for about $10^{5}$ yrs. In Fig. 1 we plot the location of each particle in the (a,e) plane at different times. The eccentricity of each single body oscillates with a period $u$ that depends on the semimajor axis of the body. If we freeze the dynamical system at subsequent times, the slow dephasing of the eccentricity of nearby orbits will translate into a wavy pattern whose density grows with time. 


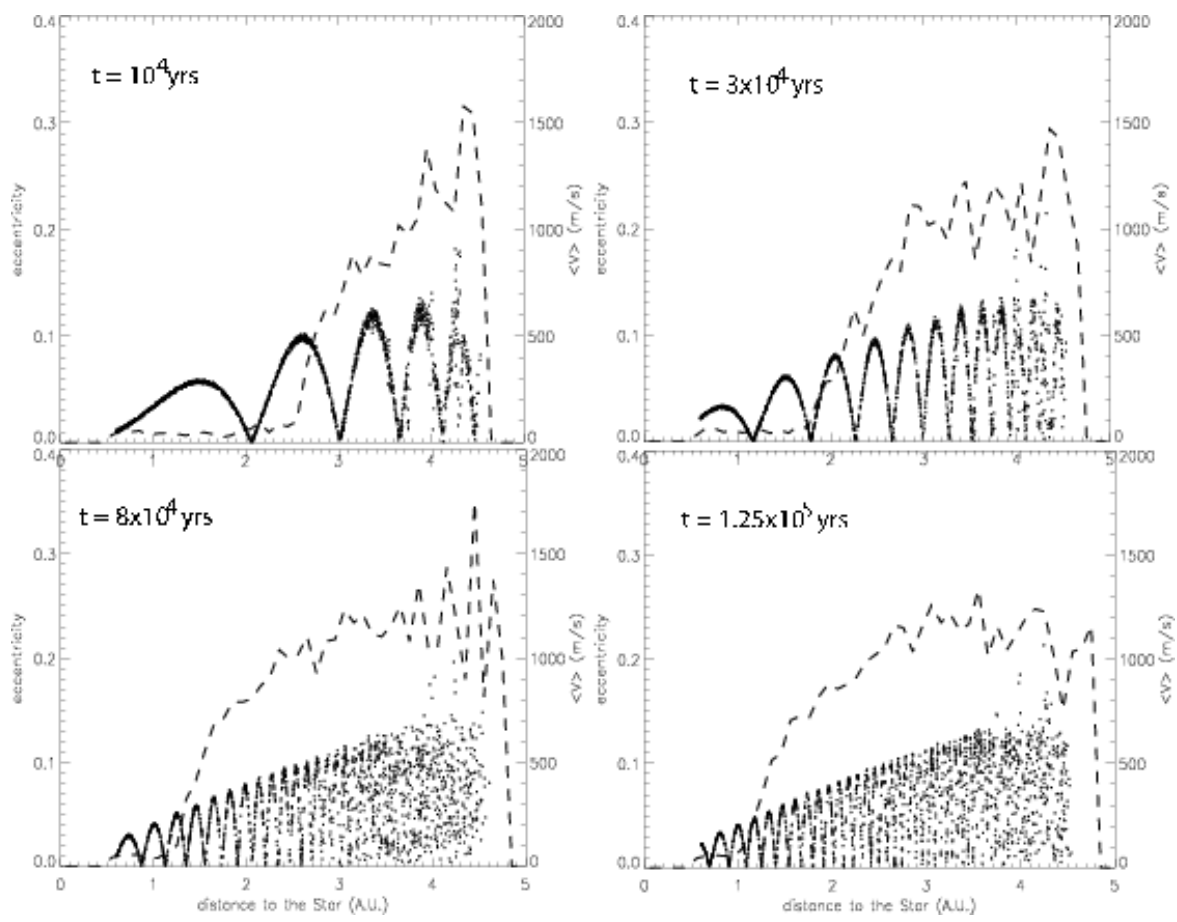

Fig. 1. Evolution of a test particle population perturbed by a stellar companion having approximately the characteristics of $\gamma$ Cephei: with $m_{b}=0.25, a_{b}=20 \mathrm{AU}$ and $e_{b}=0.3$. The dotted line represents the distribution of average encounter velocities between the bodies of the system.

\section{Effects of the secular perturbations on $\langle\Delta v\rangle$}

If the orientations of the perihelia were fully randomized in the planetesimal swarm, the effect of the secular perturbations of the companion star on $\langle\Delta v\rangle$ would be easy to derive through the standard relation (Lissauer \& Stewart, 1993):

$$
\langle\Delta v\rangle \simeq(5 / 4)^{1 / 2} e_{F} v_{K}
$$

where $v_{K}$ is the local Keplerian velocity. According to this equation, even very low values of forced eccentricity would halt accretion. In the $\gamma$ Cephei binary system, for example, $\langle\Delta v\rangle$ would be approximately $1.8 \times 10^{3} \mathrm{~m} \cdot \mathrm{s}^{-1}$ at $2 \mathrm{AU}$, the distance from the star where the planet has been found. This large relative velocity would certainly prevent mutual accretion in any swarm of kilometer-sized planetesimals (which have escape velocities of the order of a few $\mathrm{m} . \mathrm{s}^{-1}$ ). In addition, a proper eccentricity component $e_{p}$ has to be added to the planetesimal ecentricities, causing oscillations of the eccentricity around $e_{F}$ with an amplitude equal to $e_{p}$, which leads to even higher impact velocities. If this was the scenario in the early phases of planetesimal accumulation in 
close binary systems, secular perturbations pose a serious threat to planetary formation. However, the predictions of Equ.5 strongly depend on the assumption that planetesimal trajectories have a randomized distribution of orbital angles. This is not true at the beginning of the dynamical evolution, because secular perturbations force of a strong phasing of the orbits. The large eccentricity oscillations induced by the companion star are in phase and associated to a very efficient periastron alignment between neighboring orbits. This configuration leads to planetesimal encounters on almost tangential trajectories and, notwithstanding the very high values of forced eccentricity, to low and accretion friendly relative velocities, dominated by the Keplerian shear.

At subsequent times, however, the dependence of the precession frequency $u$ of $\varpi$ on $a^{1 / 2} \Delta a$ causes a progressive dephasing of nearby orbits. At some point neighbouring orbits eventually cross (see the detailed discussion in Thébault et al. (2006)), leading to a sudden and very sharp increase of the encounter velocities. This is illustrated in Fig.1 where we have superimposed to the (e,a) graph the corresponding evolution of the average relative velocity $\langle\Delta v\rangle$. The transition between the inner regions, where the orbital phasing is still strong preventing orbital crossing, and the outer regions where orbits already cross is very abrupt: $\langle\Delta v\rangle$ changes from a few $\mathrm{m} . \mathrm{s}^{-1}$ to about $1 \mathrm{~km} . \mathrm{s}^{-1}$, an accretion-inhibiting value for kilometer-sized planetesimals, within $\simeq 0.1 \mathrm{AU}$. Thébault et al. (2006) have derived an analytical estimate of the location in semimajor axis $a_{\text {cross }}$ where the orbital crossing occurs as a function of time:

$$
C_{1} a_{\text {cross }}^{1 / 2}\left[\left(\frac{a_{\text {cross }}}{a_{b}}\right)^{2}+C_{2}\left(\frac{a_{\text {cross }}}{a_{b}}\right)^{4}\right]=\frac{1}{t},
$$

where $a_{b}, e_{b}$ and $m_{b}$ are the binary semimajor axis, eccentricity and mass ratio, respectively, and:

$$
C_{1}=\frac{45 \pi}{16} \frac{e_{b}}{\left(1-e_{b}^{2}\right)^{5 / 2}} m_{b} \frac{1}{a_{b}^{2}} ; C_{2}=\frac{224}{3} \frac{m_{b}}{\left(1-e_{b}^{2}\right)^{3}} .
$$

Conversely, for a given location $a$ in the planetesimal swarm, the time $t_{c r}$ required by the wavefront of high impact speeds to reach $a_{\text {cross }}$ is:

$$
t_{c r}=6.98 \times 10^{2} \frac{\left(1-e_{b}^{2}\right)^{3}}{e_{b}}\left(\frac{m_{b}}{M_{\odot}}\right)^{1.1}\left(\frac{a_{b}}{10 \mathrm{AU}}\right)^{4.3}\left(\frac{a_{\text {cross }}}{1 \mathrm{AU}}\right)^{-2.8} \mathrm{yr} .
$$

These analytical formulae hold for $a_{b}>10 \mathrm{AU}$ and for $0.05<e_{b}<0.8$. In Fig. 2 we show the time required by the high speed regime to reach $1 \mathrm{AU}$ for for different values of $a_{b}$ and $e_{b}$ and for $m_{b}=0.5$. Note that for low $e_{b}$ and large $a_{b}, t_{c r}$ is longer than $10^{5} \mathrm{yr}$, giving a chance to runaway growth to build up large protoplanets before an erosion regime is established. 


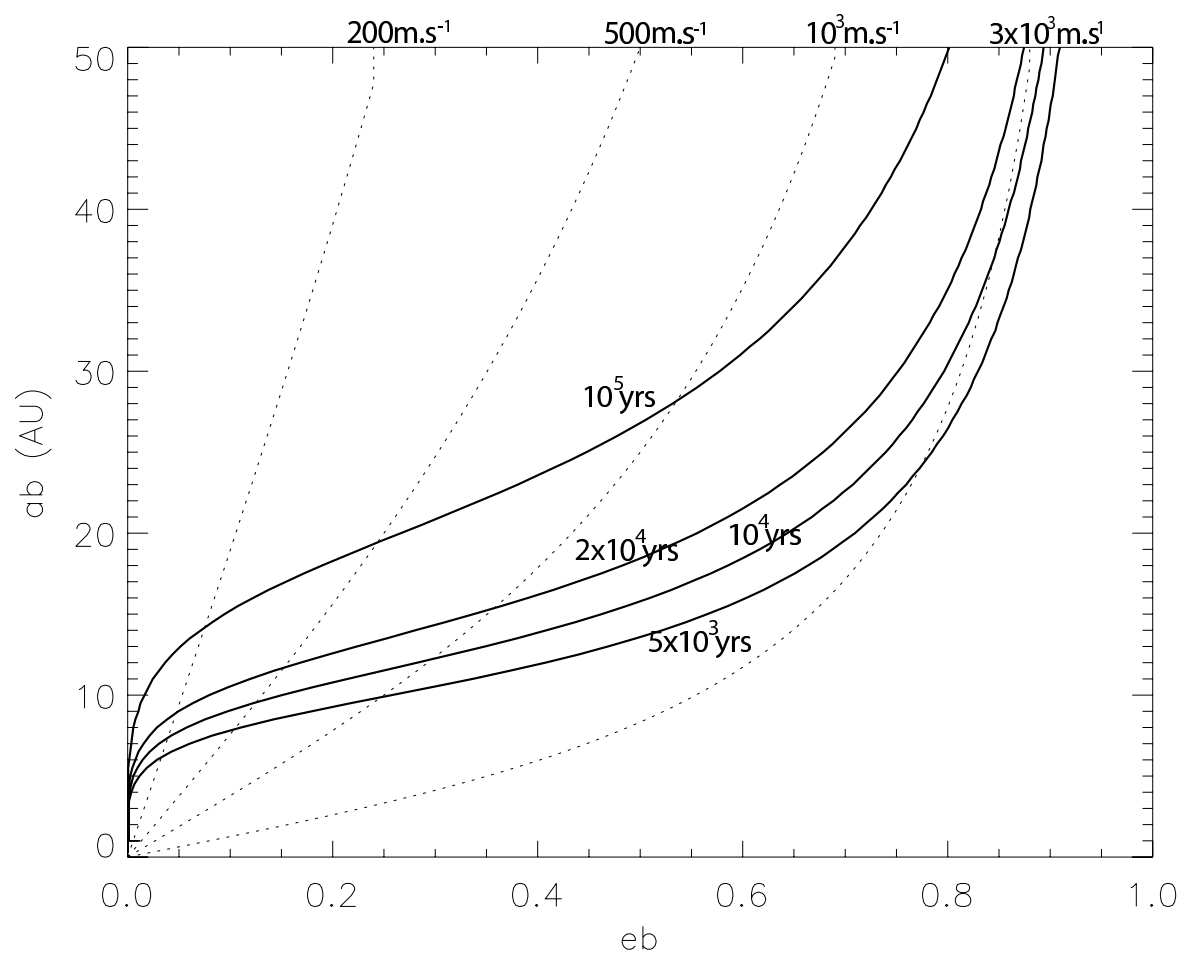

Fig. 2. Value of the minimum companion semi-major axis, $a_{b . c r 1}$, leading to orbital crossing of planetesimals at $1 \mathrm{AU}$, for different values of the crossing time $t_{c r}$, as a function of the companion eccentricity. The companion's mass is fixed, with $m_{b}=$ 0.5 . The dotted lines represent constant values of $\langle\Delta v\rangle$ at orbital crossing, as given by Equ. 5

An implicit assumption of this scenario is that the initial encounter velocities in the planetesimal swarm are very low, i.e. that the initial orbits are either circular or fully phased. This assumption is commonly adopted when addressing planetesimal accretion in binaries (e.g. Heppenheimer, 1978; Whitmire et al., 1998; Thébault et al., 2004), but should however be handled with care. Its validity depends on how and how fast planetesimals decouple from the gas of the protoplanetary disk to follow their own Keplerian orbits around the main star. If the decoupling occurs almost at the same time for all the planetesimals and if they are at that moment on almost circular orbits, then their proper eccentricities $e_{p}$ 's are approximately all equal to $e_{F}$ and the $\varpi$ 's are grouped around 0 with consequent low relative velocities. Basically, it all goes down to how the "initial" kilometer-sized planetesimals are being formed. Unfortunately, so far there are no clues on how planetesimals form in a disk perturbed by the gravity of the secondary star and how these perturbations may affect their trajectories as they emerge from the gas of the 
disk. As a matter of fact, there is not even full agreement on how planetesimals form around "normal" quiet single-stars. This very difficult exceeds by far the scope of the present, but there are schematically two competing models for planetesimal formation in a quiescent protoplanetary disk: gravitational instability (e.g. Goldreich \& Ward, 1973; Youdin \& Shu, 2002; Youdin \& Chiang, 2004), where kilometer-sized bodies directly form from small solids in a dense unstable solid grain layer in the midplane of the protoplanetary disk, and collisional-chemical sticking (e.g. Weidenschilling, 1980; Dominik \& Tielens, 1997; Dullemond \& Dominik, 2005), in which planetesimals are the outcome of a progressive mutual grain sticking. If gravitational instability works out also for disks in binary systems, then the planetesimal formation timescale is likely to be negligible compared to the typical timescales for runaway growth and for the onset of fully dephased secular perturbations by the companion star. In this case, it is reasonable to expect an initial orbital alignment within the swarm. The decoupling from the gas occurs at a slower rate in the chemical sticking scenario where planetesimals grow progressively. They might begin to feel the companion star perturbations when they are not yet fully detached from gas streamlines. However, numerical simulations indicate that the whole growth process from grains to kilometer-sized bodies might not exceed a few $10^{3}$ years (Weidenschilling, 2000), i.e. still shorter than both runaway growth and secular perturbations timescales.

\section{Role of gas drag}

So far, all effects other than the gravitational perturbations of the companion star have been neglected. However, in the standard planetary formation scenario, the initial stages of planetesimal accumulation are believed to take place in presence of significant amounts of primordial gas. Marzari and Scholl (2000) have shown that gas drag damps planetesimal eccentricities and also restores a strong perihelion alignment for equal size bodies. If the density of the gas is large enough all the $\varpi s$ of planetesimals halt their circulation and tends towards a fixed value $\varpi_{a l}$ equal to $270^{\circ}$. This tends to cancel out the large eccentricity oscillations forced by the companion. In Fig. 3 we show this phenomenon for $5 \mathrm{~km}$ planetesimals embedded in a protostellar disk similar to the minimum mass solar nebula and perturbed by an outer star. The parameters of the binary system recall those of $\alpha$ Centauri. In this simulation and in all the following ones, the drag force is modelled in laminar gas approximation as

$$
\ddot{\mathbf{r}}=-K v_{\text {rel }} \mathbf{v}_{\text {rel }},
$$

where $K$ is the drag parameter given by $K=3 \frac{\rho_{g} C_{d}}{8 \rho_{p l} s}$ (Kary et al., 1993) where $s$ is the radius of the planetesimal, $\rho_{p} l$ its mass density, $\rho_{g}$ the gas density of 
the protoplanetary disk and $C_{d}$ a dimensionless drag coefficient related to the shape of the body $(\sim 0.4 \text { for spherical bodies })^{6}$

The orbits of the planetesimals are all aligned for an indefinite time up to about $2 \mathrm{AU}$ from the star and the $\langle\Delta v\rangle$ is of the order of a few meters per second. Under this condition, planetesimal collisions lead to accretion all the time.

Unfortunately, this alignment depends on the balancing between the drag force and the strength of the secular perturbations. For a lower gas density or for a larger size of the planetesimals the perihelia moves away from $\varpi_{a l}$ towards larger values and the alignment is perturbed by a wavy pattern that forewarns the onset of a regime dominated by secular perturbations. This behaviour is illustrated in Fig.4 for a bimodal planetesimal population characterized by two different sizes.

The different alignment of planetesimals according to their size has a crucial impact on the accretion process. While equal size planetesimals keep a low $\langle\Delta v\rangle$ since their orbits are oriented towards the same angle, when different size planetesimals encounter each other the relative velocity is large most of the time because of the forced unpaired orbital alignment. The high eccentricities induced by the secular perturbations introduce a large non-tangential component in the relative velocity that easily overcomes the Keplerian shear and cause high velocity impacts. In Tables I and II we report the average encounter velocities $<\Delta v\left(R_{1}, R_{2}\right)>$ between planetesimals of size $R_{1}$ and $R_{2}$ for two different binary star orbital configurations. The assumed density of the gas drag corresponds to that of the Minimum Mass Solar Nebulae derived by Hayashi (1981). The velocity values have been obtained with numerical integrations of the planetesimal trajectories over $2 \times 10^{4}$ years. For the case

\footnotetext{
${ }^{6}$ Our gas drag model is a simplified one where the gas disk is assumed to be fully axisymmetric and follows a classical Hayashi (1981) power law distribution. It is however more than likely that in reality the gas disk should depart from this simplified view because it would also "feel" the companion star's perturbations. Several numerical studies have investigated the complex behaviour of gaseous disks in binary systems. They all show that pronounced spiral structures rapidly form within the disk (e.g. Artymowicz and Lubow, 1994; Savonije et al., 1994) and that gas streamlines exhibit radial velocities. To follow the dynamical behaviour of planetesimals in such non-axisymmetric gas profiles would require a study of the coupled evolution of both gas and planetesimal populations, have to rely on hydro-code modeling of the gas in addition to $\mathrm{N}$-body type models for the planetesimals. Such an all-encompassing gas+planetesimals modeling is clearly the next step in binary disk studies and have already been started by several teams. It is interesting to note that preliminary results seem to show that planetesimal behaviours in systems with "realisitic" gas disk modeling do not seem to drastically depart from the behaviour in the axisymmetric case. There is in particular no phase alignment between eccentric planetesimal orbits and gas streamlines, so that gas friction on planetesimals is still very high (Paardekooper, private communication).
} 

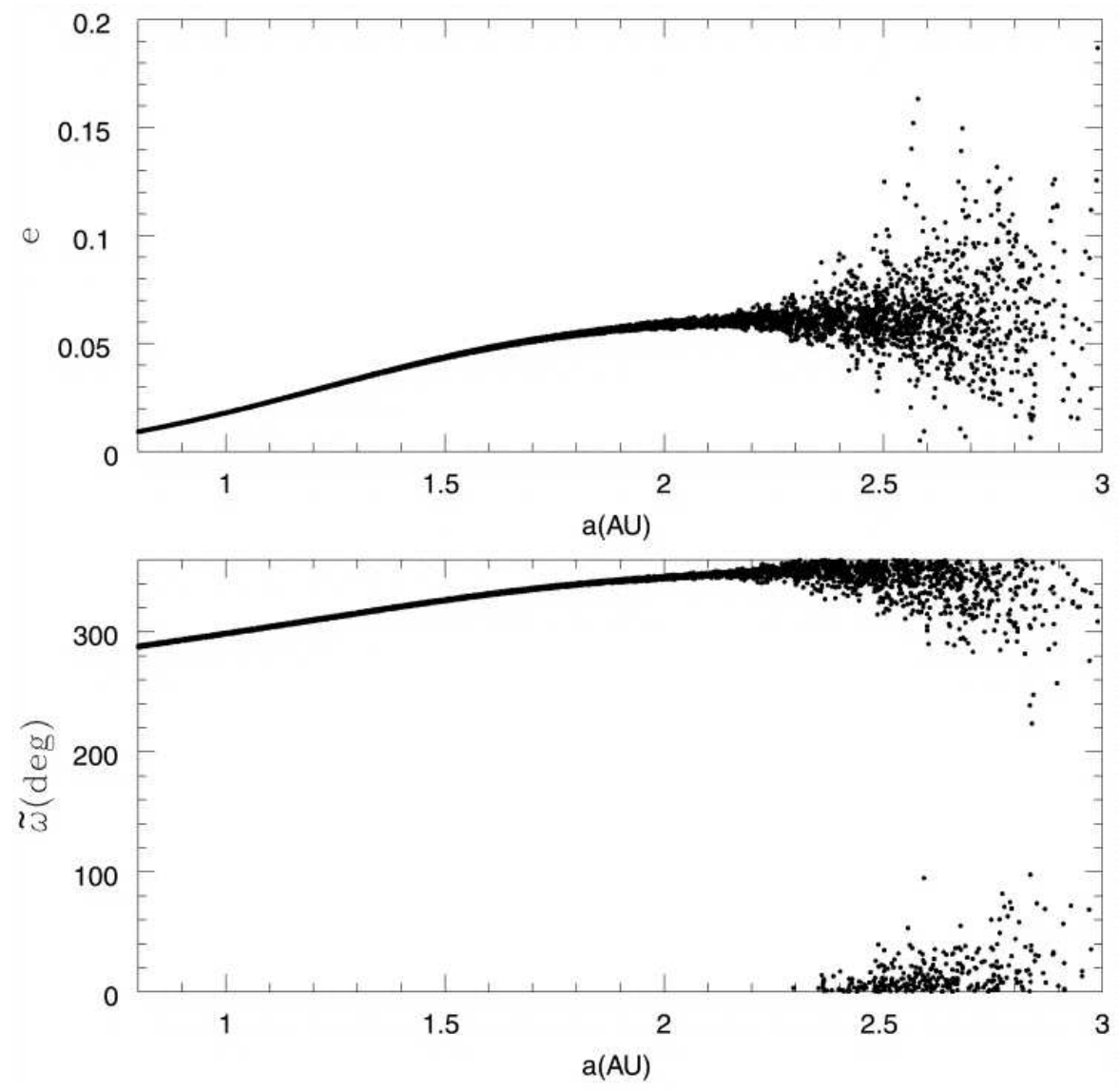

Fig. 3. Periastron and eccentricity distributions of a swarm of $5 \mathrm{~km}$ diameter planetesimals surrounding the main star of the Alpha Centauri system. After $10^{4}$ yrs from the beginning of the simulation the planetesimal orbits are strongly aligned because of the coupling between gas drag and secular perturbations of the companion star.

displayed in Table I, it is worth noticing that, in absence of the gaseous component, the parameters of the binary system would lead to orbital crossing of nearby planetesimal orbits in less than $\simeq 5 \times 10^{3}$ years (see Fig.2). The swarm would stop growing and erosion would take place. When we include the effects of gas drag the $\langle\Delta v\rangle$ remains always low for equal size planetesimals. However, for bodies of different sizes, the different phasing caused by the coupling between gas drag and secular perturbations pump up the relative velocities. 

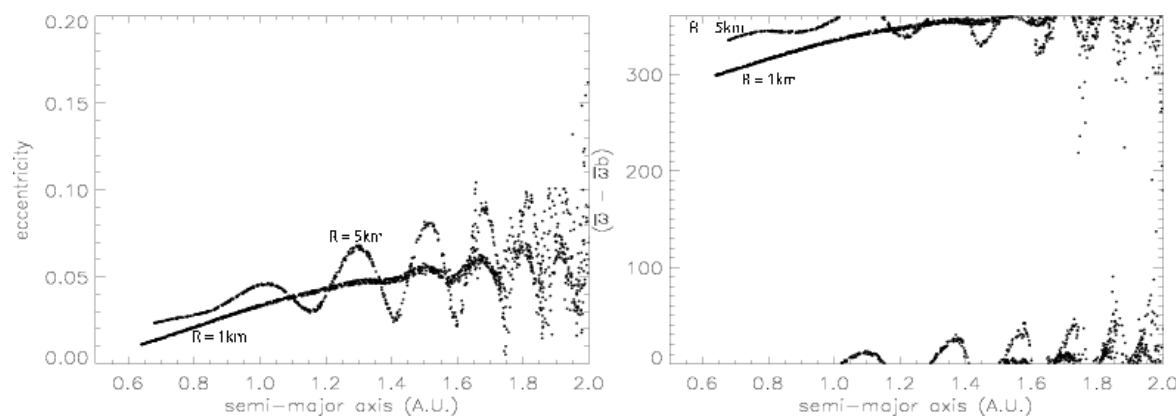

Fig. 4. Example gas drag run. Snapshots, at $t=3 \times 10^{3} \mathrm{yrs}$, of the $(e, a)$ and $\left(\varpi-\varpi_{b}, a\right)$ distributions for 2 planetesimal populations of size $R_{1}=1 \mathrm{~km}$ and $R_{2}=5 \mathrm{~km} . \varpi-\varpi_{b}$ is the difference, in angular degrees, between the particles and the companion star's longitude of periastron. The companion star orbital parameters are: $a_{b}=10 \mathrm{AU}, e_{b}=0.3, m_{b}=0.5$

Table 1. Average encounter velocities in $\mathrm{m} . \mathrm{s}^{-1}$, at $1 \mathrm{AU}$ from the primary, within a population of "small" planetesimals $1<R<10 \mathrm{~km}$ for a gas drag simulation with the companion star parameters $m_{b}=0.5, a_{b}=10 \mathrm{AU}$ and $e_{b}=0.3 m_{b}=0.5$, $a_{b}=20 \mathrm{AU}$ and $e_{b}=0.4 . \Delta v_{R 1, R 2}$ values are averaged over the time interval $0<$ $t<2 \times 10^{4}$ yrs. Initial starting encounter velocities are such as $\Delta v_{0} \simeq 10 \mathrm{~m}$. s. $^{-1}$. $\Delta v$ values in bold correspond to accreting impacts for all tested collision outcome prescriptions. Underlined values are those for which we obtain different accretion vs. erosion balance depending on the tested prescription. Values in classical roman characters correspond to cases for which all tested models agree on a net erosive outcome.

\begin{tabular}{c|cccccccccc} 
& \multicolumn{10}{|c}{} \\
\hline Sizes $(\mathrm{km})$ & 1 & 2 & 3 & 4 & 5 & 6 & 7 & 8 & 9 & 10 \\
\hline 1 & $\mathbf{1 0}$ & 154 & 233 & 285 & 327 & 360 & 391 & 426 & 452 & 458 \\
2 & 172 & $\mathbf{1 0}$ & 94 & 133 & 187 & 223 & 262 & 287 & 316 & 334 \\
3 & 238 & 84 & $\mathbf{1 1}$ & 54 & 99 & 137 & 177 & 200 & 230 & 254 \\
4 & 289 & 144 & 63 & $\mathbf{1 2}$ & $\underline{40}$ & 80 & 115 & 149 & 171 & 198 \\
5 & 325 & 188 & 103 & $\underline{43}$ & $\mathbf{1 2}$ & $\underline{32}$ & $\underline{70}$ & 100 & 122 & 154 \\
6 & 373 & 228 & 144 & 83 & $\underline{32}$ & $\mathbf{1 1}$ & $\mathbf{3 6}$ & $\underline{56}$ & 84 & 104 \\
7 & 400 & 261 & 182 & 113 & $\underline{68}$ & $\mathbf{3 6}$ & $\mathbf{1 2}$ & $\mathbf{3 5}$ & $\underline{48}$ & $\underline{76}$ \\
8 & 428 & 298 & 212 & 147 & 98 & $\underline{56}$ & $\mathbf{3 5}$ & $\mathbf{1 2}$ & $\mathbf{3 6}$ & $\underline{45}$ \\
9 & 450 & 310 & 238 & 168 & 123 & 83 & $\underline{48}$ & $\mathbf{3 6}$ & $\mathbf{1 3}$ & $\mathbf{3 1}$ \\
10 & 453 & 338 & 263 & 196 & 152 & 107 & $\underline{73}$ & $\underline{48}$ & $\mathbf{3 1}$ & $\mathbf{1 3}$
\end{tabular}


Table 2. Same as Table 1 , but with companion star parameters $m_{b}=0.5, a_{b}=$ $20 \mathrm{AU}$ and $e_{b}=0.4$.

\begin{tabular}{c|cccccccccc} 
& \multicolumn{10}{|c}{} \\
\hline Sizes $(\mathrm{km})$ & 1 & 2 & 3 & 4 & 5 & 6 & 7 & 8 & 9 & 10 \\
\hline 1 & $\mathbf{1 1}$ & 127 & 204 & 255 & 298 & 342 & 368 & 390 & 417 & 442 \\
2 & 126 & $\mathbf{1 0}$ & 84 & 139 & 185 & 227 & 258 & 290 & 317 & 340 \\
3 & 200 & 91 & $\mathbf{1 1}$ & 68 & 108 & 158 & 186 & 218 & 246 & 272 \\
4 & 258 & 146 & 61 & $\mathbf{9}$ & $\underline{48}$ & 88 & 120 & 154 & 186 & 209 \\
5 & 301 & 192 & 113 & 54 & $\mathbf{1 2}$ & $\underline{44}$ & $\underline{75}$ & 111 & 136 & 164 \\
6 & 339 & 232 & 152 & 99 & $\underline{43}$ & $\mathbf{1 0}$ & $\mathbf{3 1}$ & $\underline{66}$ & 92 & 119 \\
7 & 361 & 262 & 181 & 126 & $\underline{77}$ & $\mathbf{2 8}$ & $\mathbf{1 3}$ & $\underline{\mathbf{2 6}}$ & $\underline{56}$ & 87 \\
8 & 395 & 295 & 219 & 159 & 112 & $\underline{68}$ & $\mathbf{2 6}$ & $\mathbf{1 1}$ & $\mathbf{2 8}$ & $\underline{48}$ \\
9 & 425 & 320 & 246 & 190 & 136 & 92 & $\underline{55}$ & $\mathbf{2 5}$ & $\mathbf{1 1}$ & $\mathbf{2 3}$ \\
10 & 446 & 346 & 266 & 208 & 163 & 122 & 82 & $\underline{49}$ & $\mathbf{2 2}$ & $\mathbf{1 2}$
\end{tabular}

\section{Dependence of the accretion process on the binary parameters.}

Once we determine the $\left\langle\Delta v_{\left(R_{1}, R_{2}\right)}\right\rangle$ for any pair of planetesimal size we must compare it with the threshold velocity $v_{\text {ero }}$ for that pair of projectile and target to test whether either erosion or accretion will be the result of the collision. As pointed out in Thébault et al. (2006), in literature one can find many prescriptions of the collisional outcome between two planetesimals given their impact velocity. Adopting the approach outlined in Thébault et al. (2006), three different values of $v_{\text {ero }}$ are computed according to Holsapple (1994), Marzari et al. (1995) and Benz \& Asphaug (1999). Erosion (or accumulation) is assumed to occur when $\left\langle\Delta v_{\left(R_{1}, R_{2}\right)}\right\rangle$ is higher (or lower) than all the three values of $v_{\text {ero }}$. In the intermediate cases we prefer not to draw any definitive conclusion letting them as undefined cases. In Tabs.1\&2 a different text font is used to indicate when the computed $\left\langle\Delta v_{\left(R_{1}, R_{2}\right)}\right\rangle$ leads to accretion, erosion, or to an undefined situation. By inspecting the tables, it is interesting to note that anytime we depart from the diagonal stripe $R_{1}=R_{2}$ there is a sharp increase of $\langle\Delta v\rangle$, almost always exceeding the threshold value for accretion, at least for the binary parameters adopted in the tables.

The knowledge of the relative velocities between different size planetesimals is the first step towards fixing constraints on the dynamical environment allowing planetary formation. These velocities should be used in the context of a full numerical model for planetesimal accretion that takes into account also the frequency with which different or equal size planetesimals collide. If the size distribution of the swarm privileges impacts between equal size planetesimals, it comes out clearly from the data in the tables that accretion is possible also for very close and eccentric binary systems. On the other hand, if collisions between different size planetesimals dominate the evolution of the planetesimal swarm, then the different alignment of perihelia might halt the accretion process. Unfortunately, the size distribution of a planetesimal swarm 
is poorly constrained at the beginning of the accretion process and it may also be a misleading concept (Wetherill \& Inaba, 2000). The frequently adopted assumption that "initial" planetesimals have a given size $R_{p l}$ is numerically convenient but certainly an oversimplification. Regardless of the details of the planetesimal formation process, there have to be a dispersion $\Delta R_{p l}$ in planetesimal sizes. The amplitude of $\Delta R_{p l}$ is difficult to estimate, but it seems likely that it spans over at least one (if not several) order(s) of magnitude (see for instance Fig.3 of Weidenschilling, 2000). If this is the case, then in the binary stars cases explored here, encounters leading to erosion ("NA" type encounters) might largely dominate over those leading to accretion (type "A"). We have checked this by performing a simplified test where we assume for the planetesimal size distribution a Gaussian function centered on $R=5 \mathrm{~km}$ of variance $\Delta R_{p l}^{2}$. By counting the number of A-type encounters vs. NA-types using the values of $\langle\Delta v\rangle_{\left(R_{1}, R_{2}\right)}$ reported in Tabs. 1 and 2 we find that accretion dominates over erosion only when $\Delta R_{p l}<0.8 \mathrm{~km}$. This value of $\Delta R_{p l}$ appears to be small, possibly too small for any realistic initial size distribution. As a consequence, gas drag tends to prevent, or at least slow down, accretion in the initial stages of planetesimal evolution. As accretion proceeds, larger bodies are formed and impacts occur more and more frequently between different size bodies. The different phasing caused by gas drag for different size planetesimals increases the number of NA-type encounters and erosion dominates, at least for the binary parameters given in the tables.

The limit of a purely dynamical approach that estimates the planetesimal relative velocity but neglects their size distribution can be partly overcome by taking into account that on average the projectiles delivering the maximum kinetic energy to a target of size $R_{2}$ are roughly those of size $R_{1} \simeq 1 / 2 R_{2}$ (see Thébault et al., 2006). With this assumption we can probe the feasibility of accretion by testing $\langle\Delta v\rangle$ against $v_{\text {ero }}$ only for a limited number of different size body pairs. By exploiting this simplification, Thébault et al. (2006) have tested the chances of planetesimal accretion for 120 different binary systems with semimajor axis $a_{B}$ ranging from 10 to $50 \mathrm{AU}$ and eccentricity from 0.05 to 0.9 . The mass ratio has been kept fixed to $m_{b}=0.5$. In all the simulations only two size pairs have been considered, $R_{1}=2.5 \mathrm{~km}$ vs. $R_{2}=5 \mathrm{~km}$, representative of small planetesimals, and $R_{1}=15 \mathrm{~km}$ vs. $R_{2}=50 \mathrm{~km}$, representative of intermediate size bodies in the swarm. In Figs.5and6 different color coding are used to outline the planetesimal collisional fate, depending on the binary parameters:

- 1) Dark green. The evolution of a planetesimal disk in binaries belonging to these regions is not significantly affected by the companion star perturbations. $\langle\Delta v\rangle$ remains low and accretion should proceed as in the standard model for single stars.

- 2) Light green. Within these regions, $\langle\Delta v\rangle$ is increased by the gas drag coupling to the secular perturbations but remains below $v_{\text {ero }}$. Accretion is still possible but the amplitude of the gravitational focusing factor 
$\left(v_{e s c\left(R_{2}\right)} / \Delta v\right)^{2}$ is significantly reduced. This slows down the accretion rate compared to the single-star unperturbed case.

- 3) Yellow. Here $\langle\Delta v\rangle$ is comparable to the erosion velocities computed with the different prescriptions adopted at the beginning of the study for $v_{\text {ero }}$. This is a limiting situation where the preference of the system towards accretion or erosion depends on the details of the still not well defined collisional physics. We prefer not to draw any definite conclusion in this case.

- 4) Orange and Red. In these regions, $\langle\Delta v\rangle$ exceeds $v_{\text {ero }}$ for all three different collision outcome prescriptions considered. Collisions always result in a net mass loss and mutual accretion is impossible.

As expected, systems with higher eccentricity or lower binary separation are more critical for planetesimal accretion. An empirical fit has been performed in Thébault et al. (2006) to outline the region in the $\left(a_{b}, e_{b}\right)$ phase space where accretion is possible for the two different size ranges of planetesimals:

$$
e_{b} \simeq 0.013\left(\frac{a_{b}}{10 \mathrm{AU}}\right)^{2} \quad \text { for small } 1<\mathrm{R}<10 \mathrm{~km} \text { planetesimals },
$$

and

$$
e_{b} \simeq 0.018\left(\frac{a_{b}}{10 \mathrm{AU}}\right)^{2}, \text { for large } 10<\mathrm{R}<50 \mathrm{~km} \text { planetesimals. }
$$

By extrapolating the fit to large values of $a_{b}$ one can figure out that for binary separations $a_{b} \geq 90 \mathrm{AU}$ the planetesimal accretion process is not significantly perturbed by the companion star gravity.

One interesting feature is the extent of the "light-green" region where planetesimal accretion is not halted but possibly slowed down since the gravitational focusing factor is reduced. As a consequence, "classical" runaway growth is not possible and might be replaced by the alternative, possibly slower type II runaway mode identified by Kortenkamp et al. (2001).

\section{Type II runaway growth}

In this section we describe details of planetesimal accretion simulations in the Sun-Jupiter "binary" system (Kortenkamp \& Wetherill, 2000a; Kortenkamp et al., 2001). This system has traditionally been used for initial characterizations of dynamical effects in binary-star systems (e.g. Heppenheimer, 1978; Whitmire et al., 1998). In the models described here we use units of binary separation $D$ rather than AU. Note also that the modeling included Saturn as well (a triple system) but we omit Saturn from most of the discussion, referring simply instead to the Sun-Jupiter "binary."

Initial orbits for the planetesimals were circular, coplanar with the nebular midplane, and uniformly distributed from $10-50 \%$ of $D$. As the system evolves 


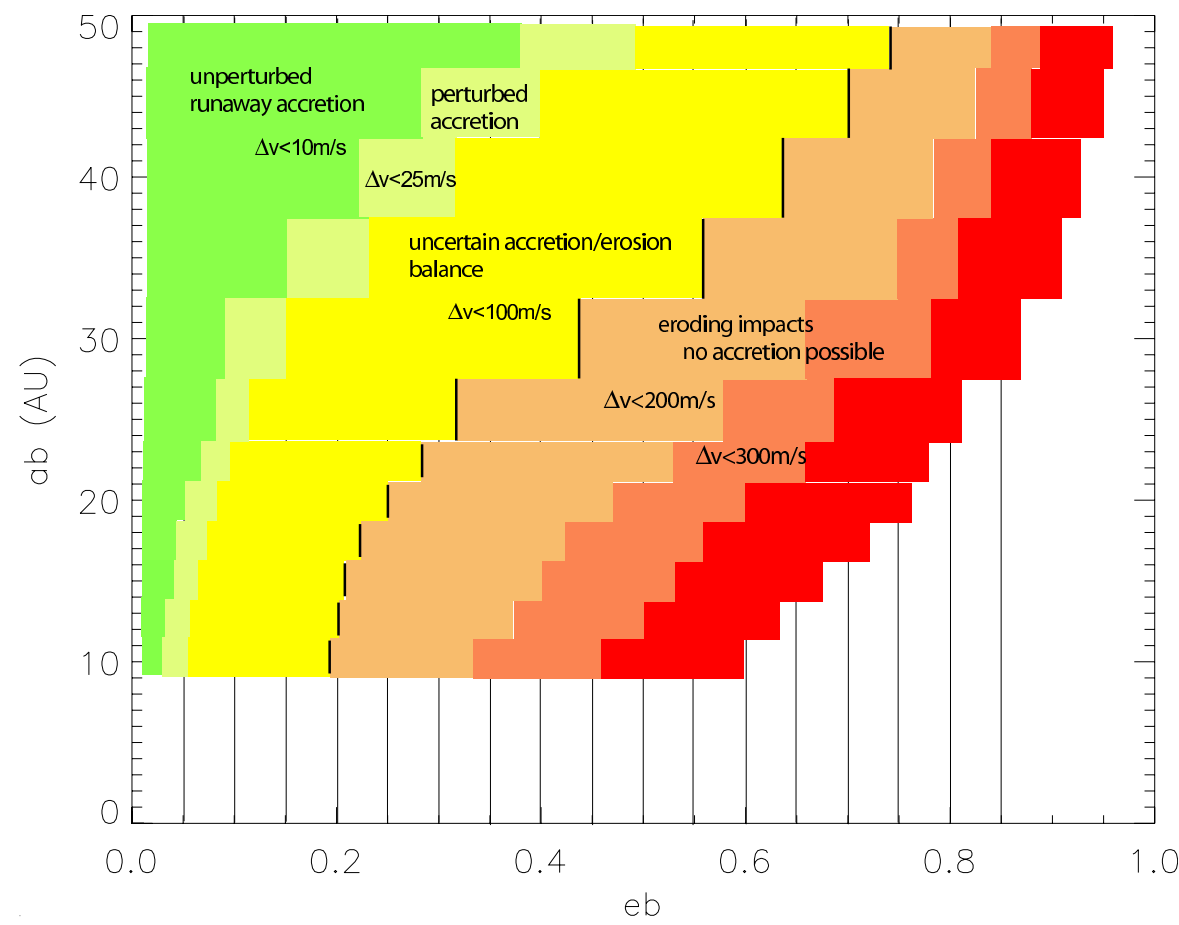

Fig. 5. Encounter velocities averaged, over the time interval $0<t<2 \times 10^{4}$ yrs, between $R_{1}=2.5$ and $R_{2}=5 \mathrm{~km}$ bodies at $1 \mathrm{AU}$ from the primary star, for different values of the companion star semi-major axis and eccentricity. The short black vertical segments mark the limit beyond which $\left\langle\Delta v_{(R 1, R 2)}\right\rangle$ values correspond to eroding impacts for all tested collision outcome prescriptions.

the combination of gas drag and secular perturbations from the companion Jupiter (and Saturn) leads to a size-dependence in $e$ and $I$ as well as in the phasing of the orbital orientation angles, as described earlier in this review chapter. Note that the system is fully three dimensional. The companion orbits are inclined with respect to each other and the protoplanetary disk.

As described above, the distribution of encounter velocities is a critical factor which determines how a population of planetesimals will accumulate into larger bodies. High encounter velocities can actually lead to impact erosion of the target rather than growth. On the other hand, low encounter velocities can gravitationally enhance the collision cross-section of the target (Öpik, 1951). The effective cross-section becomes

$$
\sigma_{\mathrm{eff}}=\sigma\left(1+\frac{v_{\mathrm{esc}}^{2}}{v_{\mathrm{enc}}^{2}}\right)
$$

where $\sigma$ is the target's geometric cross-section, $v_{\text {enc }}$ is the projectile's encounter velocity, and $v_{\text {esc }}$ is the combined surface escape velocity of the target and projectile. 


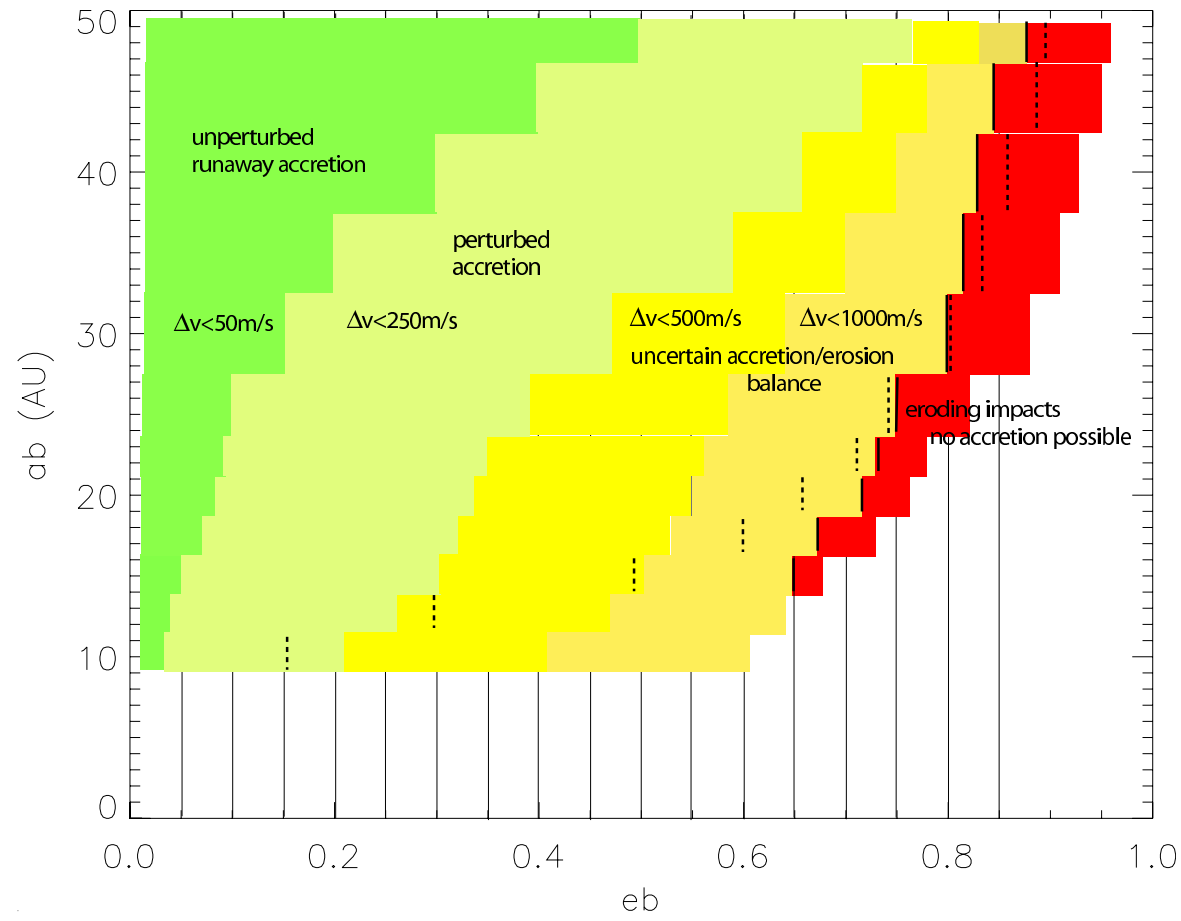

Fig. 6. Same as Fig.5, but for $R_{1}=15$ and $R_{2}=50 \mathrm{~km}$ bodies. The short black vertical segments mark the limit beyond which $\left\langle\Delta v_{(R 1, R 2)}\right\rangle$ values correspond to eroding impacts for all tested collision outcome prescriptions. The short dashed vertical segments mark the position beyond which orbital crossing occurs, despite the effects of gas drag, for $50 \mathrm{~km}$ bodies.

The size-dependent phasing of orbital elements leads to low encounter velocities between similar size bodies and high encounter velocities between bodies of markedly different size. For the Sun-Jupiter model described above "binary" encounter velocities were calculated for all intersecting orbits over the entire range of planetesimal sizes. Figure 7 shows examples of these en- 


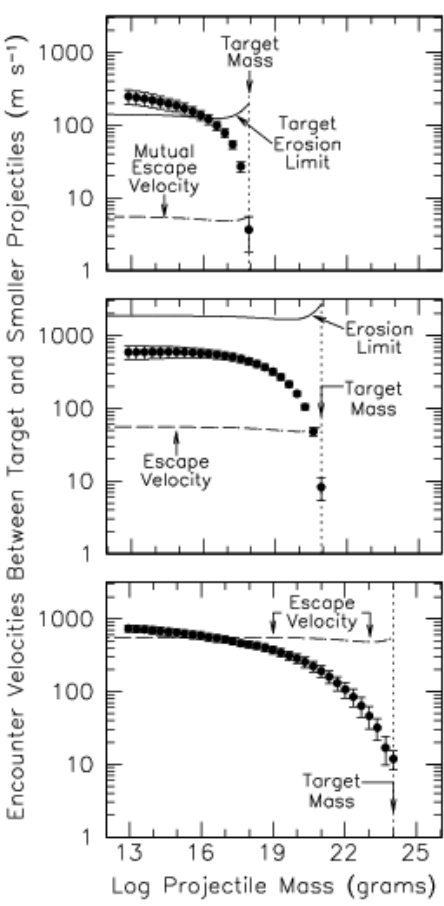

counter velocities for various target bodies and equal or lesser size projectile bodies with orbits near $33 \% D$, where, again, $D$ is the Sun-Jupiter separation distance.

Modeling the growth of planetesimals in this scenario is considerably more difficult than tracking their orbital evolution. Full-scale $N$-body simulations of planetesimal growth that include mutual gravitational perturbations, secular perturbations, and gas drag are beyond the reach of current techniques. Theoretically one would need to include $\sim 10^{12}$ small $\left(\sim 10^{14} \mathrm{~g}\right)$ planetesimals to form a single $10^{26} \mathrm{~g}$ terrestrial planet embryo. Direct $N$-body integrations of mutually perturbing planetesimals cannot even remotely approach this figure, treating only about $\sim 10^{4}$ bodies over the time scale required. On the other hand, existing statistical simulations of planetary growth are not limited by the number of bodies. However, these simulations assume that the orbits are completely randomized so they cannot easily accommodate the size-dependent orbital evolution described above. To overcome these shortcomings, Kortenkamp et al. (2001) developed a hybrid approach that capitalizes on the strengths of each technique. They used $N$-body integration to map the size-dependent velocity distributions. These velocity distributions are then used in modified statistical accretion simulations to model the collisional accumulation of the planetesimals. This approach led to the identification of 


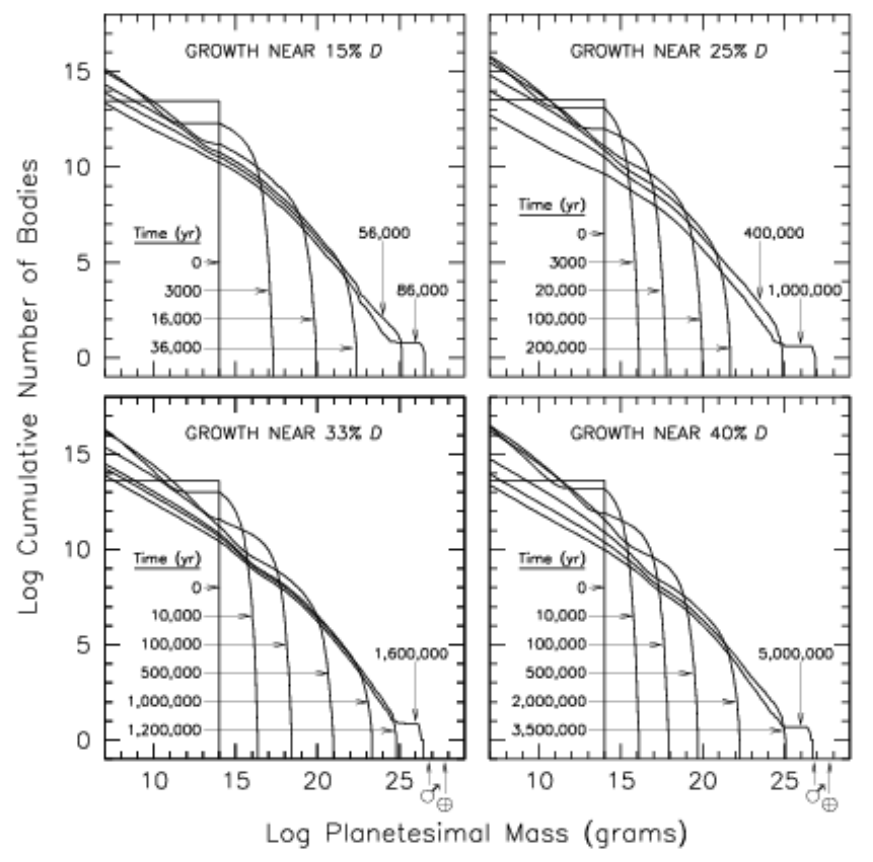

Fig. 8. Growth of planetesimals in a circumsolar disk perturbed by the "companion" Jupiter at a distance $D$ from the sun. The habitable zone, simply defined here as the Venus-Mars region, stretches from about $10-25 \%$ of $D$. Initially all planetesimals have identical masses of $10^{14}$ grams. The initial surface density of planetesimals at $15 \%$ of $D$ is $10 \mathrm{~g} \mathrm{~cm}^{-2}$, scaling as $r^{-3 / 2}$ with heliocentric distance $r$. This is roughly consistent with the so-called "minimum mass" surface density. Collision fragments smaller than $10^{7} \mathrm{~g}(\sim 1$ meter $)$ are presumed lost via nebular gas drag and therefore removed from the simulation. Growth is calculated in four different regions centered on 15, 25, 33, and $40 \%$ of $D$. For comparison, the masses of Earth and Mars are indicated by their respective symbols. The top two panels represent growth in the habitable zone and show initial orderly growth transitioning to Type II runaway growth of Mars-size planetary embryos in $10^{5}$ to $10^{6}$ years. Beyond the habitable zone (bottom panels) growth is slower but eventually also produces Marssize embryos. Figure is adapted from Kortenkamp et al. (2001).

Four separate simulations were performed, centered on $15 \%, 25 \%, 33 \%$ and $40 \%$ of $D$. For comparison, the habitable zone extends from about 10 $25 \%$ of $D$ (crudely defined as the Venus-Mars region). In all four regions the growth is characterized as "orderly" (non-runaway, see Safronov, 1969) until the distribution reaches approximately $10^{24} \mathrm{~g}$, or about the size of the 
largest asteroid-(1) Ceres. The planetesimal size distribution then becomes bi-modal, transitioning to runaway growth and producing Mars-size embryos. All planetesimals were assumed to be uniformly distributed across a region and any bodies separated by more than 10 mutual Hill radii were considered dynamically isolated (Chambers et al., 1996) and not allowed to collide. This resulted in multiple runaway embryos emerging in each region.

Note that mutual perturbations between planetesimals were not included in these calculations. This new form of runaway growth arises when secular perturbations and gas drag act together to establish size-dependent encounter velocities that remain low when colliding bodies are of similar size. Collisions between bodies of significantly different size are at high velocity and can lead to cratering and erosion, but these simulations show that growth overcomes erosion (Fig. 8). This alternative mode of growth, which was eloquently dubbed "Type II" runaway growth, allowed for the formation of terrestrial planet embryos throughout the habitable zone of the Sun-Jupiter "binary." This general result should apply regardless of whether the perturbations are from Jupiter-like companions formed earlier by disk-instability (e.g. Boss, 1997; Mayer et al., 2002), stellar-mass objects in multiple-star systems, or perhaps even short-lived instabilities that lead to asymmetries in protoplanetary disks.

Classical "Type I" runaway growth occurs in a self-gravitating population of planetesimals. Random orbital kinetic energy is exchanged during gravitational encounters between large and small bodies and the population trends towards energy equipartition, a process dubbed "dynamical friction" (Stewart \& Kaula, 1980). Dynamical friction lowers the encounter velocities of the larger bodies with respect to each other, enhancing their effective collision cross-sections and increasing the rate at which they accumulate each other. Under these conditions nearly the entire growth period up to embryo-size is in Type I runaway mode. In our simulations, which are not self-gravitating, the size-dependent phasing of orbital elements holds encounter velocities low between all similar-size bodies (typically 1 to $10 \mathrm{~m} \mathrm{~s}^{-1}$, see Fig. 7). Initially these encounter velocities exceed the planetesimal escape velocities so there is no enhancement of collision cross-sections and growth is orderly. As larger and larger bodies grow their escape velocities approach and then exceed the relatively low encounter velocities, causing the transition from orderly growth to Type II runaway growth. In this way, the effects of dynamical friction are mimicked by the size-dependent phasing of orbital elements.

\section{Conclusions}

The process of planetary formation around the primary star of a binary system is complicated in all its stages by the gravitational perturbations of the companion star. However, the existence of some gas giant planets in binary systems with separation of a few tens of AU suggests that these perturba- 
tions are not always strong enough to prevent the formation of a planet. We have analysed within the standard model of planet growth how the secular perturbations of the companion star affect the various stages of planetesimal accretion. Ours studies show that when we include also the effects of gas drag on the motion of small planetesimals, their orbits become phased both in eccentricity and perihelion longitude (Marzari \& Scholl, 2000). At a first sight, this alignment favors fast accretion keeping low the encounter velocities between the bodies in spite of the large values of eccentricity forced by the secular perturbations. However, the angle towards which the perihelia align depends on the size of the planetesimals and on their distance from the star (Thébault et al., 2004, 2006). In the earlier stages of planetesimal accretion all the bodies have roughly the same size and the encounter velocities, because of the favorable phasing, are low. However, as soon as larger bodies emerge in the population the different alignments of the orbits lead to large impact velocities that may prevent the onset of runaway growth or even cause erosion of the bodies inhibiting the formation of a planet. Through a full numerical approach we have analysed to which extent the different alignment influences the random planetesimal velocities. We have also mapped the values of the binary orbital parameters (semi-major axis and eccenticity) for which accumulation dominates over erosion despite of the secular perturbations, or viceversa. Our modelling shows that for binary of separations $a_{b} \leq 40 \mathrm{AU}$, only very low $e_{b}$ allow planetesimal accretion to proceed as in the standard single-star case. On the contrary, only relatively high $e_{b}$ values (of at least 0.2 in the closest $a_{b}=10 \mathrm{AU}$ sepration explored and at least $\sim 0.7$ for $a_{b}=40 \mathrm{AU}$ ) lead to a complete stop of planetesimal accretion. In most cases, when the perturbations of the massive companion on the planetesimal disk is significant but not strong enough to halt accretion, runaway growth can still occur, but in a different way respect to the classical "Type I" runaway typical of planetesimal populations around single stars. This new type of growth termed "Type II" runaway allows planet formation to occur in binary-star systems with much tighter orbits than previously suggested (Heppenheimer, 1978; Whitmire et al., 1998). However, there is (at least) one caveat. As already noted, the growth simulations described above and represented in Figure 8 did not include mutual perturbations of the planetesimals themselves. Crude attempts at including self-gravitating planetesimals (Kortenkamp \& Wetherill, 2000b) indicated that when the size distribution reaches $10^{24}$ to $10^{25} \mathrm{~g}$ the mutual perturbations are beginning to become important, although they are still dominated by the size-dependent phasing of secular perturbations. This suggests that just as Type II runaway growth is getting underway accretion may either stall or perhaps transition to the classical Type I runaway growth. To explore these possibilities we are working to modify the multi-zone planetesimal accretion code of Weidenschilling et al. (1997) to include secular perturbations from massive companions as well as mutual perturbations from planetesimals and nebular gas drag (Kortenkamp et al., 2006). 


\section{Acknowledgments}

S. Kortenkamp acknowledges support from NASA for some of this work under grants NNG04GP56G and NNG04GI14G.

\section{References}

Adachi, I., Hayashi, C., Nakagawa, K., 1976, The gas drag effect on the elliptical motion of a solid body in the primordial solar nebula, Prog. Theor. Phys., 56, 1756

Artymowicz, P., Lubow, Stephen H., 1994, Dynamics of binary-disk interaction. 1: Resonances and disk gap sizes, ApJ, 421, 651

Barge, P., Pellat, R., 1993, Mass spectrum and velocity dispersions during planetesimal accumulation, Icarus, 104, 79

Benz, W., Asphaug, E., 1999, Catastrophic Disruptions Revisited, Icarus, 142, 5

Bodenheimer, P., Lin, D. N. C., 2002, Implications of Extrasolar Planets for Understanding Planet Formation Annual Review of Earth and Planetary Sciences 30, 113-148.

Boss, A. P., 1997, Giant planet formation by gravitational instability. Science, 276, 1836-1839

Chambers, J. E., Wetherill, G. W., 1998, Making the Terrestrial Planets: NBody Integrations of Planetary Embryos in Three Dimensions, Icarus 136, 304-327.

Chambers J.E., Wetherill G.W., Boss A.P., 1996, The stability of multi-planet systems, Icarus, 119, 261.

Chen, C. H., Kamp, I., 2004, ApJ 602, 985-992.

Cuzzi, J., Weidenschilling, S., Particle-Gas Dynamics and Primary Accretion, 2006, in Meteorites and the Early Solar System II, D. S. Lauretta and H. Y. McSween Jr. (eds.), University of Arizona Press, Tucson, 943 pp., p.353-381

Desidera, S., Barbieri, M., 2007, A\&A 462, 345-353

Dominik, C.; Tielens, A., The Physics of Dust Coagulation and the Structure of Dust Aggregates in Space, 1997, ApJ, 480, 647

Dullemond, C., Dominik, C., Dust coagulation in protoplanetary disks: A rapid depletion of small grains, 2005, A\&A, 434, 971

Duquennoy, A.; Mayor, M., 1991, Multiplicity among solar-type stars in the solar neighborhood. II-Distribution of the orbital elements in an unbiased sample, A\&A, 248, 485

Eggenberger, A., Udry, S., Mayor, M., 2003, in ASP Conf. Ser. 294, Scientific Frontiers in Research on Extrasolar Planets, ed. D. Denning \& S. Seager, 43

Goldreich, P., Ward, W., 1973, The Formation of Planetesimals, ApJ, 183, 1051 
Greenberg, R.; Hartmann, W. K.; Chapman, C. R.; Wacker, J. F., 1978, Planetesimals to planets - Numerical simulation of collisional evolution, Icarus, 35,1

Guillot, T., 1999, A comparison of the interiors of Jupiter and Saturn, Planetary and Space Science 47, 1183-1200.

Haisch, K. E., Lada, E. A., Lada, C. J., 2001, ApJ 553, L153-L156.

Hatzes, A. P., Cochran, W. D., Endl, M., McArthur, B., Paulson, D. B., Walker, G. A. H., Campbell, B., Yang, S., 2003, A Planetary Companion to Gamma Cephei A, ApJ. 599, 1383-1394.

Hayashi, C., 1981, Structure of the solar nebula, growth and decay of magnetic fields and effects of magnetic and turbulent viscosities on the nebula, PthPS 70,35

Heppenheimer, T., 1978, On the formation of planets in binary star systems, A\&A 65,421

Holsapple, K. A., 1994, Catastrophic disruptions and cratering of solar system bodies: A review and new results, P\&SS, 42, 1067

Kary, D. M., Lissauer, J. J., Greenzweig, Y., 1993, Nebular gas drag and planetary accretion, Icarus 106, 288-307.

Kokubo, Eiichiro; Ida, Shigeru, 1998, Oligarchic Growth of Protoplanets, Icarus, 131, 171

Kokubo, Eiichiro; Ida, Shigeru, 2000, Formation of Protoplanets from Planetesimals in the Solar Nebula, Icarus, 143, 15

Kortenkamp S.J., Wetherill G.W., 2000a, Terrestrial planet and asteroid formation in the presence of giant planets I. Relative velocities of planetesimals subject to Jupiter and Saturn perturbations, Icarus, 143, 60.

Kortenkamp S.J., Wetherill G.W., 2000b, Formation of the asteroid belt. Lunar Plan. Sci. Conf., 31, abstract \#1813.

Kortenkamp S.J., Kokubo E., Weidenschilling S.J., 2000, Formation of planetary embryos, In Origin of the Earth and Moon, Canup R.M., Righter K., Eds. (Univ. Arizona Press, Tucson), pp. 85-100.

Kortenkamp, S., Wetherill, G., Inaba, S., 2001, Runaway Growth of Planetary Embryos Facilitated by Massive Bodies in a Protoplanetary Disk, Science, 293, 1127

Kortenkamp S.J., Weidenschilling S.J., Marzari F., 2006, A new code for modeling planetesimal accretion in protoplanetary disks perturbed by massive companions $38^{\text {th }}$ DPS Meeting, Pasadena, CA, abstract \#63.03.

Lagrange A.-M., Beust H., Udry S., Chauvin G. and Mayor M. , 2006, New constrains on Gliese 86 B - VLT near infrared coronographic imaging survey of planetary hosts, A\&A. 459, 955.

Lissauer, J.J., 1993, Planet formation, ARA\&A 31, 129

Lissauer J., Stewart G., 1993, Growth of planets from planetesimals, in Protostars and Planets III, the Univ. of Arizona Press, Tucson, 1061

Marzari, F.; Davis, D.; Vanzani, V., 1995, Collisional evolution of asteroid families, Icarus, 113, 168 
Marzari F., Scholl H., 1998, Capture of Trojans by a Growing Proto-Jupiter, Icarus, 131, 41

Marzari F., Scholl H., 2000, Planetesimal Accretion in Binary Star Systems, ApJ 543, 328

Mayer L., Quinn T., Wadsley J., Stadel J., Formation of giant planets by fragmentation of protoplanetary disks, Science, 298, 1756.

Mugrauer, M., and Neuhauser, R., 2005, Gl 86B: a white dwarf orbits an exoplanet host star, MNRAS 361, L15.

Nelson, A., 2000, Planet Formation is Unlikely in Equal-Mass Binary Systems with $a \simeq 50 \mathrm{AU}, \mathrm{ApJ}, 537,65$

Neuhäuser, Ralph; Mugrauer, Markus; Fukagawa, Misato; Torres, Guillermo; Schmidt, Tobias, 2007, Direct detection of exoplanet host star companion gamma Cep B and revised masses for both stars and the sub-stellar object, A\&A, in press, eprint arXiv:astro-ph/0611427

Öpik E.J., 1951, Collision probabilities with the planets and the distribution of interplanetary matter, Proc. Irish Acad. 54, 165.

Papaloizou, J. and Lin, D. N. C., 1984, On the tidal interaction between protoplanets and the primordial solar nebula. I - Linear calculation of the role of angular momentum exchange, ApJ 285, 818-834.

Pollack, J. B., Hubickyj, O., Bodenheimer, P., Lissauer, J. J., Podolak, M., Greenzweig, Y., 1996, Formation of the Giant Planets by Concurrent Accretion of Solids and Gas, Icarus 124, pp. 62-85.

Rafikov, R. 2003, The Growth of Planetary Embryos: Orderly, Runaway, or Oligarchic?, AJ, 125, 942

Rafikov, R. 2004, Fast Accretion of Small Planetesimals by Protoplanetary Cores, AJ, 128, 1348

Raghavan, Deepak; Henry, Todd J.; Mason, Brian D.; Subasavage, John P.; Jao, Wei-Chun; Beaulieu, Thom D.; Hambly, Nigel C., 2006, Two Suns in The Sky: Stellar Multiplicity in Exoplanet Systems, ApJ, 646, 523

On the Formation Timescale and Core Masses of Gas Giant Planets, ApJ 598, L55-L58.

Safronov, V.S., 1969, Evolution of the Protoplanetary Cloud and Formation of the Earth and the Planets. Israel program for scientific translation, TT-F 677

Santos, N. C., Israelian, G., Mayor, M., 2004, Spectroscopic [Fe/H] for 98 extra-solar planet-host stars. Exploring the probability of planet formation, A\&A 415, 1153-1166.

Savonije, G. J., Papaloizou, J. C. B., Lin, D., 1994, On Tidally Induced Shocks in Accretion Disks in Close Binary Systems, MNRAS, 268, 13

Stewart G.R., Kaula W.M., 1980, Gravitational kinetic theory for planetesimals, Icarus, 44, 154.

Strom, S. E., Edwards, S., Skrutskie, M. F., Evolutionary time scales for circumstellar disks associated with intermediate- and solar-type stars. in Protostars and Planets III, University of Arizona Press, Tucson, p. 837866 . 
Thébault, P., Marzari, F., Scholl, H., 2002, Terrestrial planet formation in exoplanetary systems with a giant planet on an external orbit, A\&A, 384, 594

Thébault, P., Brahic, A. 1998, Dynamical influence of a proto-Jupiter on a disc of colliding planetesimals, P\&SS, 47, 233

Thébault, P., Beust, H., 2001, Falling evaporating bodies in the 0̆3b2 Pictoris system. Resonance refilling and long term duration of the phenomenon, A\&A, 376, 621

Thébault P., Augereau, J.-C., Beust, H., 2003, Dust production from collisions in extrasolar planetary systems. The inner beta Pictoris disc, A\&A, 408, 775

Thébault, P., Marzari, F., Scholl, H., Turrini, D., Barbieri, M.,2004, Planetary formation in the $\gamma$ Cephei system, A\&A, 427, 1097

Thébault, P., Marzari, F., Scholl, H., 2006, Relative velocities among accreting planetesimals in binary systems: The circumprimary case, Icarus, 183, 193

Torres, G., 2007, The Planet Host Star $\gamma$ Cephei: Physical Properties, the Binary Orbit, and the Mass of the substellar companion, ApJ, 654, 1095

Weidenschilling, S., 1977, The distribution of mass in the planetary system and solar nebula, Astrophysics and Space Science 51, 153-158.

Weidenschilling, S., 1980,Dust to planetesimals- Settling and coagulation in the solar nebula, Icarus, 44, 172

Weidenschilling, S. J, 2000,Formation of Planetesimals and Accretion of the Terrestrial Planets, SSRv, 92, 295

Weidenschilling and Davis, D. R., 1985, Orbital resonances in the solar nebula - Implications for planetary accretion, Icarus, 62,16

Weidenschilling S.J., Spaute D., Davis D.R., Marzari F., Ohtsuki K., Accretional evolution of a planetesimal swarm II: The terrestrial zone. Icarus 128, 429.

Wetherill, G.W., Stewart, G.R., 1989, Accumulation of a swarm of small planetesimals, Icarus, 77, 330

Wetherill, G.W., Inaba, S., 2000, Planetary Accumulation with a Continuous Supply of Planetesimals, SSRv, 92, 311

Whitmire, D., Matese, J., Criswell, L., 1998, Habitable Planet Formation in Binary Star Systems, Icarus, 132, 196

Wuchterl, G., Guillot, T. and Lissauer, J. J., 2000, Giant Planet Formation, in Protostars and Planets IV, Mannings, V., Boss, A.P., Russell, S. S. (eds.) University of Arizona Press, Tucson, p. 1081

Youdin, A., Shu, F., 2002, Planetesimal Formation by Gravitational Instability, ApJ, 580, 494

Youdin, A., Chiang, E., 2004, Particle Pileups and Planetesimal Formation, ApJ, 601, 1109

Zucker, S.; Mazeh, T.; Santos, N. C.; Udry, S.; Mayor, M., 2004, Multi-order TODCOR: Application to observations taken with the CORALIE echelle spectrograph. II. A planet in the system HD 41004, 2004, A\&A, 426, 695 


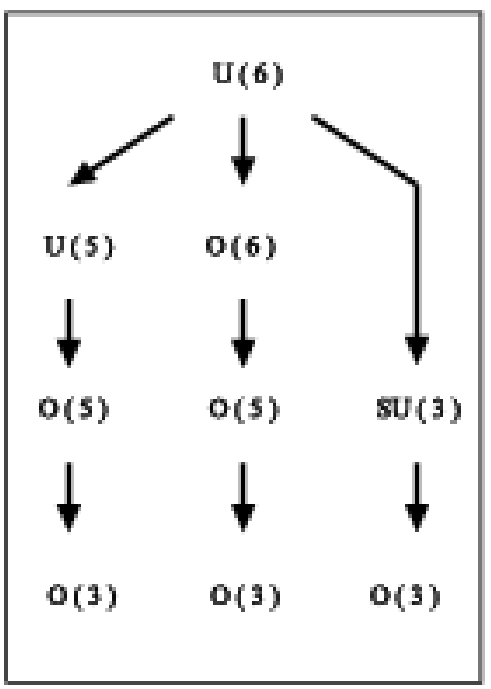

